\title{
Cystic dilatation of umbilical cord associated with patent urachus
}

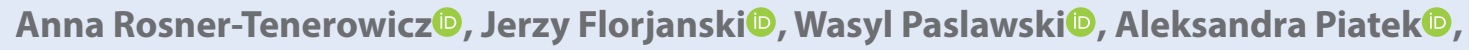 \\ Mariusz Zimmer(D) \\ $2^{\text {nd }}$ Department of Gynecology and Obstetrics, Wroclaw Medical University, Wroclaw, Poland
}

\begin{abstract}
An urachus is a remnant of allantois in embryo development constituting a connection between the dome of the bladder and umbilicus. It develops on day 16 after conception and closes during the pregnancy to form the median umbilical ligament. Patent urachus results from a failure in closing its lumen in 10-12 gestational weeks. This anomaly occurs in 1-2 babies in 100,000 births. We present the case of patent urachus. In 20 gestational weeks, a dilatation of the umbilical cord with an anechoic mass with a transverse dimension of $19 \times 12 \mathrm{~mm}$ starting from the fetal insertion and length of $30 \mathrm{~cm}$ was seen on ultrasound without any other fetal and placental disorders. Histology showed cystic edema. Prenatal diagnosis of patent urachus can be difficult because this pathology may be mistaken with other, more dangerous causes of cord cysts; thus, the occurrence of cord cysts should be closely monitored.
\end{abstract}

Key words: patent urachus; prenatal diagnosis; giant umbilical cord

Ginekologia Polska 2021; 92, 8: 595-596

\section{INTRODUCTION}

An urachus is a remnant of allantois in embryo development constituting a connection between the dome of the bladder and umbilicus. The patent urachus is a result of a failure in lumen closing in the $10^{\text {th }}-12^{\text {th }}$ week. It is a rare anomaly with the incidence of $1-2$ in 100,000 births [1].

\section{CASE REPORT}

We present the case of a 35 -year-old patient, gravida 4 para 3 referred to the hospital in the $20^{\text {th }}$ week of pregnancy because of a dilatation of the umbilical cord with an anechoic mass of the dimension of $19 \times 12 \mathrm{~mm}$ in transverse view (Fig. 1A) starting from the fetal insertion and extending on the length of around $30 \mathrm{~cm}$ (Fig. 1B). The umbilical arteries and veins were noted to be separate from the cystic mass. Pulsed wave Doppler of the umbilical vessels revealed normal indices for gestational age and no flow inside the cystic structure (Fig. 1C). No other fetal anomalies were seen. The amniotic fluid volume was in the normal range and fetal biometry was consistent with dates. Prenatal examination in the first trimester was normal.

The patient's obstetrics history included one spontaneous abortion in the $8^{\text {th }}$ week of gestation and two natural deliveries of healthy babies. Medical history during gestation included pregnancy-induced hypertension detected in the $33^{\text {rd }}$ week, urinary tract infection in the $29^{\text {th }}$ weekend, and candidiasis of the vagina. Serologic evaluation of the mother was negative for infection (TORCH).

The male infant was born at term by Cesarean section due to premature rupture of membranes and failed induction of labor. The Apgar score was 10, and the birth weight was $3940 \mathrm{~g}$. Three-vessel umbilical cord had $50 \mathrm{~cm}$ in length, and histological testing showed cystic edema in the length of $30 \mathrm{~cm}$ with a maximum diameter of the umbilical cord of $5 \mathrm{~cm}$ (Fig. 1D).

The diagnosis confirmed patent urachus and was not associated with other anomalies. After delivery, the patent urachus was ligated by surgeons. The postoperative course was uneventful. 


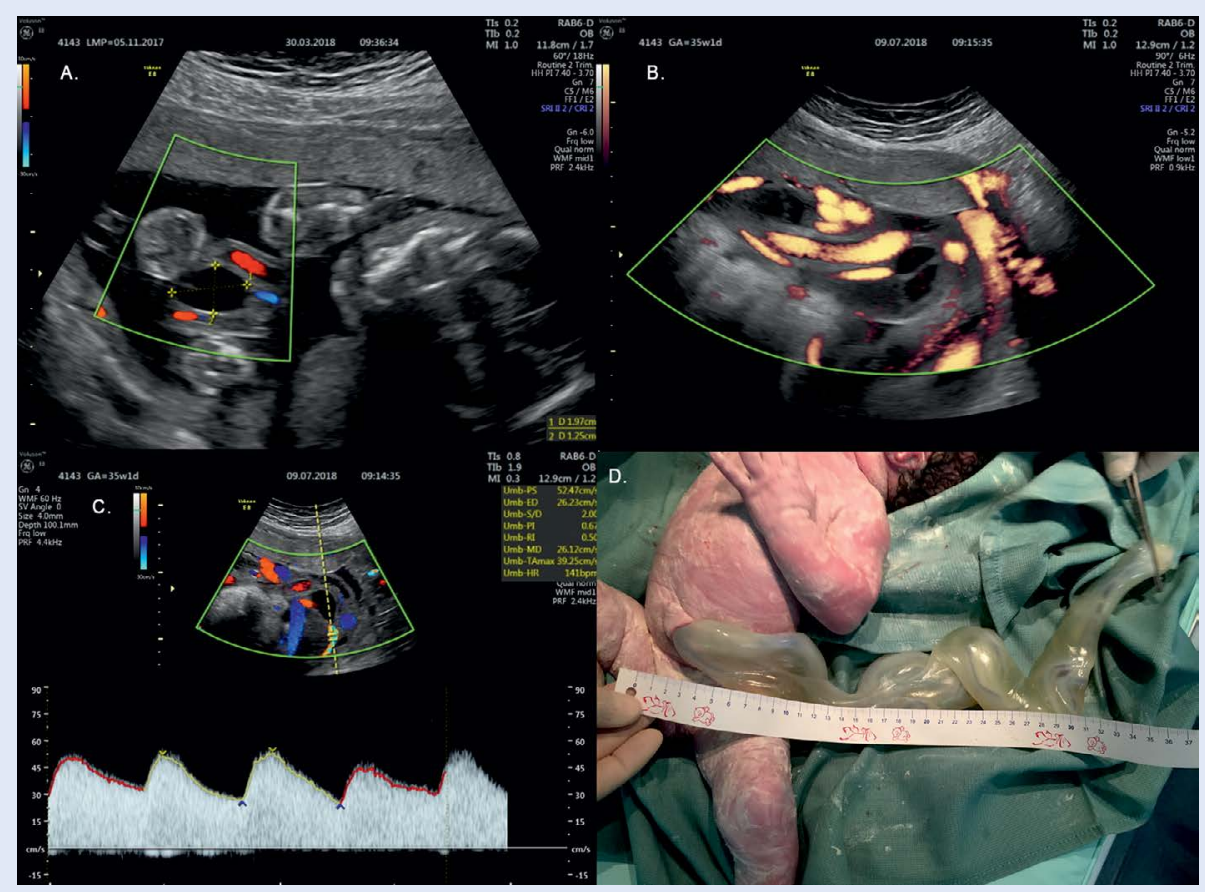

Figure 1. Characteristics of patent urachua; A. The anechoic mass of the umbilical cord in the transverse view; B. An anechoic mass originating from the fetal insertion and extending to the length of around $30 \mathrm{~cm} ; \mathbf{C}$. Pulsed wave Doppler of the umbilical vessels showing normal indices for gestational age and no flow inside the cystic structure; $\mathbf{D}$. Cystic edema measuring $30 \mathrm{~cm}$ with a maximum diameter of the umbilical cord of $5 \mathrm{~cm}$

\section{DISCUSSION AND CONCLUSIONS}

The giant umbilical cord is a pathognomonic sign of patent urachus associated with Wharton's jelly edema. The antenatal diagnosis might be made based on ultrasonographic examination revealing a communication between the bladder and cystic umbilical mass [1]. The differential diagnosis of a giant umbilical cord comprises umbilical cord pseudocysts, umbilical hernias, umbilical bladder extrophy, abdominal wall defects, vascular malformations, urachal anomalies and omphalomesenteric duct remnants [2]. In cases of patent urachus-associated giant umbilical cord, the size of the umbilical cord is much larger than in umbilical pseudocysts [2].

Although, in most of the reported cases, babies had a good prognosis, the patent urachus might lead to the intrauterine fetal demise [3]. Also, a strong association of umbilical cord cystic masses and chromosomal anomalies or congenital malformations has been found, but especially if detected in the second or third trimester. Those diagnosed in the first trimester usually disappear and have no impact on the fetal development [4].

Each case of cord cysts should be associated with close fetal monitoring and intensified surveillance. With higher awareness of patent urachus and proper diagnosis, parents may avoid emotional distress and unnecessary medical interventions.

\section{Conflict of interests}

The authors declare that they have no competing interests.

\section{REFERENCES}

1. Schiesser M, Lapaire O, Holzgreve W, et al. Umbilical cord edema associated with patent urachus. Ultrasound Obstet Gynecol. 2003; 22(6): 646-647, doi: 10.1002/uog.924, indexed in Pubmed: 14689541.

2. Nobuhara KK, Lukish JR, Hartman GE, et al. The giant umbilical cord: an unusual presentation of a patent urachus. J Pediatr Surg. 2004; 39(1): 128-129, doi: 10.1016/j.jpedsurg.2003.09.023, indexed in Pubmed: 14694391.

3. Brooks K, Guileyardo J, Le D, et al. Giant cystic umbilical cord associated with patent urachus and intrauterine fetal demise. Proc (Bayl Univ Med Cent). 2017; 30(2): 209-210, doi: 10.1080/08998280.2017.11929589, indexed in Pubmed: 28405085.

4. Sepulveda W, Wong AE, Gonzalez R, et al. Fetal death due to umbilical cord hematoma: a rare complication of umbilical cord cyst. J Matern Fetal Neonatal Med. 2005; 18(6): 387-390, doi: 10.1080/14767050500277602, indexed in Pubmed: 16390804. 